\title{
Topological Pressure for One-Dimensional Holomorphic Dynamical Systems
}

\author{
by
}

\section{Katrin GELFERT and Christian WOLF}

\author{
Presented by Stanistaw KWAPIEŃ
}

Summary. For a class of one-dimensional holomorphic maps $f$ of the Riemann sphere we prove that for a wide class of potentials $\varphi$ the topological pressure is entirely determined by the values of $\varphi$ on the repelling periodic points of $f$. This is a version of a classical result of Bowen for hyperbolic diffeomorphisms in the holomorphic non-uniformly hyperbolic setting.

1. Introduction. In this paper we study the topological pressure $P_{\text {top }}(\varphi)$ $=P_{\text {top }}(f, \varphi)$ of a continuous potential $\varphi$ with respect to a one-dimensional holomorphic dynamical system $f$. To simplify the exposition we discuss in the introduction exclusively the case when $f$ is a rational map of the Riemann sphere and present our more general results later on. Let $f: \overline{\mathbb{C}} \rightarrow \overline{\mathbb{C}}$ be a rational map of degree $d \geq 2$, and let $J$ denote the Julia set of $f$, i.e. the closure of the repelling periodic points of $f$ (see [2] for details). We are interested in the topological pressure with respect to the dynamical system $f \mid J$.

We denote by $\operatorname{Per}_{n}(f)$ the fixed points of $f^{n}$ in $J$ and by $\operatorname{Per}(f)=$ $\bigcup_{n} \operatorname{Per}_{n}(f)$ the periodic points of $f$ in $J$. Moreover, let $\operatorname{Per}_{\text {rep }}(f) \subset \operatorname{Per}(f)$ denote the set of repelling periodic points of $f$. Given $\alpha>0,0<c \leq 1$, and $n \in \mathbb{N}$ we define

$$
\begin{aligned}
\operatorname{Per}_{n}(\alpha, c)=\left\{z \in \operatorname{Per}_{n}(f):\left|\left(f^{k}\right)^{\prime}\left(f^{i}(z)\right)\right|\right. & \geq c \exp (k \alpha) \\
\text { for all } k & \in \mathbb{N} \text { and } 0 \leq i \leq n-1\} .
\end{aligned}
$$

2000 Mathematics Subject Classification: 37F10, 37D25, 37D35, 28D20.

Key words and phrases: topological pressure, rational maps, holomorphic dynamics, repelling periodic points, invariant measures.

The research of K.G. was supported by the grant EU SPADE2. She is grateful to IMPAN for the hospitality. 
Thus, if $\alpha \geq \alpha^{\prime}, c \geq c^{\prime}$, then

$$
\operatorname{Per}_{n}(\alpha, c) \subset \operatorname{Per}_{n}\left(\alpha^{\prime}, c^{\prime}\right)
$$

and

$$
\operatorname{Per}_{\text {rep }}(f)=\bigcup_{\alpha>0} \bigcup_{c>0} \bigcup_{n=1}^{\infty} \operatorname{Per}_{n}(\alpha, c)
$$

Let $\mathcal{M}$ denote the set of all $f$-invariant Borel probability measures on $J$ endowed with the weak* topology. This makes $\mathcal{M}$ into a compact convex space. Moreover, let $\mathcal{M}_{\mathrm{E}} \subset \mathcal{M}$ be the subset of ergodic measures. For $\mu \in \mathcal{M}_{\mathrm{E}}$ we define the Lyapunov exponent of $\mu$ by

$$
\chi(\mu)=\int \log \left|f^{\prime}\right| d \mu .
$$

It follows from Birkhoff's ergodic theorem that the pointwise Lyapunov exponent at $z$, which is defined by

$$
\chi(z)=\lim _{n \rightarrow \infty} \frac{1}{n} \log \left|\left(f^{n}\right)^{\prime}(z)\right|,
$$

exists for $\mu$-a.e. $z \in J$ and coincides (whenever it exists) with $\chi(\mu)$. We say that a measure $\mu$ is hyperbolic if $\chi(\mu)>0$. We denote by $h_{\mu}(f)$ the measure-theoretic entropy of $f$ with respect to $\mu$ (see for example [15] for the definition). Moreover, we denote by $P_{\text {top }}(\varphi)$ the topological pressure of $\varphi$ with respect to $f$ (see Section 2.2 for the definition). For $\varphi \in C(J, \mathbb{R})$ we define

$$
\alpha(\varphi)=\sup \left\{\chi(\mu): \mu \in \mathcal{M}_{\mathrm{E}} \cap \operatorname{ES}(\varphi)\right\}
$$

where $\operatorname{ES}(\varphi)$ denotes the set of equilibrium states of $\varphi$, i.e. of measures $\mu \in \mathcal{M}$ satisfying $P_{\text {top }}(\varphi)=h_{\mu}(f)+\int \varphi d \mu\left(^{1}\right)$. We note that it follows from a general result of Newhouse [10] (or alternatively from a theorem of Lyubich [9] or from Freire et al. [5] in the case of rational maps) that for all $\varphi \in C(J, \mathbb{R})$ we have $\operatorname{ES}(\varphi) \cap \mathcal{M}_{\mathrm{E}} \neq \emptyset$. Our main goal in this paper is to prove the following result (for a more general case of not necessarily rational maps see Theorem 2 below):

TheOREM 1. Let $f: \overline{\mathbb{C}} \rightarrow \overline{\mathbb{C}}$ be a rational map of and let $\varphi \in C(J, \mathbb{R})$ be a Hölder continuous potential.

(a) If $\alpha(\varphi)>0$ then for all $0<\alpha<\alpha(\varphi)$ we have

$$
P_{\text {top }}(\varphi)=\lim _{c \rightarrow 0} \limsup _{n \rightarrow \infty} \frac{1}{n} \log \left(\sum_{z \in \operatorname{Per}_{n}(\alpha, c)} \exp \left(\sum_{k=0}^{n-1} \varphi\left(f^{k}(z)\right)\right)\right) .
$$

$\left({ }^{1}\right)$ Note that the supremum in (5) is in fact a maximum. This follows from the fact that $\operatorname{ES}(\varphi)$ is a non-empty compact convex set whose extremal points are precisely the ergodic measures. 
(b) If (6) is true for some $\alpha>0$ then there exists an ergodic equilibrium state $\mu$ of $\varphi$ with $\chi(\mu) \geq \alpha$.

We note that Theorem 1 generalizes a well-known result of Bowen for Axiom A diffeomorphisms to the case of holomorphic non-uniformly expanding dynamical systems. For a related result in the case of non-uniformly hyperbolic diffeomorphisms we refer to [6].

We briefly mention work where related assumptions on the potentials have been used. Note that the prerequisite of Theorem 1 is satisfied if $P_{\text {top }}(\varphi)>\max _{z \in J} \varphi(z)$ and in particular if $\max _{z \in J} \varphi(z)-\min _{z \in J} \varphi(z)<$ $h_{\text {top }}(f \mid J)$ are satisfied, which are (much stronger than $\alpha(\varphi)>0$ ) open conditions in the $C^{0}$ topology. The latter condition has been mentioned first in [7] in the context of piecewise monotonic maps of the unit interval and of a bounded variation potential $\varphi$ to guarantee the existence and good ergodic properties of equilibrium states for $\varphi$, using a spectral gap approach. In [4], it is shown that for a rational map of degree $\geq 2$ on the Riemann sphere for a Hölder continuous potential $\varphi$ satisfying $P_{\text {top }}(\varphi)>\sup \varphi$, there is a unique equilibrium state for $\varphi$. Analogous results are obtained for a class of non-uniformly expanding local diffeomorphisms and Hölder continuous potentials satisfying such a low oscillation condition (see [1] and references therein).

Przytycki et al. [12] consider a pressure of the potential $-t \log \left|f^{\prime}\right|$ which is defined as in (6) except that they use all periodic points rather than only points in $\operatorname{Per}_{n}(\alpha, c)$. They prove the equality between this pressure and various other types of pressures in the case of rational maps satisfying an additional hypothesis that not too many periodic orbits with Lyapunov exponent close to 1 move close together (which is satisfied if $f$ is a topological Collet-Eckmann map or, equivalently, if $f$ is uniformly expanding on periodic orbits). It would be interesting to know under which conditions their pressure coincides with the pressure in (6).

This paper is organized as follows. In Section 2 we introduce a class of one-dimensional holomorphic (not necessarily rational) dynamical systems and discuss various notions of topological pressure. In Section 3 we prove our main result showing that for this class of systems the topological pressure is entirely determined by the values of the potential on the repelling periodic points.

\section{Preliminaries}

2.1. A class of one-dimensional holomorphic dynamical systems. Let $X \subset \overline{\mathbb{C}}$ be compact and let $f: X \rightarrow X$ be continuous. We say that $f \in \mathcal{A}(X)$ if there is an open neighborhood $U$ of $X$ such that $f$ extends to a holomorphic 
map on $U$ and for every $z \in U \backslash X$, either $z$ leaves $U$ under iteration of $f$, or $\liminf _{n \rightarrow \infty} \frac{1}{n} \log \left|\left(f^{n}\right)^{\prime}(z)\right|=0$.

Without further mention we will always use a specific set $U$ associated with $X$ and $f$ and we will also denote the extension of $f$ to $U$ by $f$. We note that in the particular case when $f$ is a rational map on $\overline{\mathbb{C}}$ with Julia set $J$, a normal family argument shows that $f \in \mathcal{A}(J)$. For $f \in \mathcal{A}(X)$ we will continue to use the notation from Section 1 (e.g. $\operatorname{Per}(f), \operatorname{Per}_{\text {rep }}(f), \operatorname{Per}_{n}(\alpha, c), \mathcal{M}, \mathcal{M}_{\mathrm{E}}$, $\chi(\mu), \chi(z), \alpha(\varphi)$, etc.) for $f \mid X$.

Let now $U \subset \overline{\mathbb{C}}$ be open and $f: U \rightarrow \overline{\mathbb{C}}$ be holomorphic. We say that $f$ is expanding on a compact $f$-invariant set $\Lambda \subset U$ if there exist constants $c>0$ and $\beta>1$ such that

$$
\left|\left(f^{n}\right)^{\prime}(z)\right| \geq c \beta^{n}
$$

for all $n \in \mathbb{N}$ and all $z \in \Lambda$. We note that for $f \in \mathcal{A}(X)$ every invariant expanding set $\Lambda \subset U$ is contained in $X$. This follows from (7).

2.2. Various pressures. We first recall the definition of the classical topological pressure. Let $(X, d)$ be a compact metric space and let $f: X \rightarrow X$ be a continuous map. For $n \in \mathbb{N}$ we define a new metric $d_{n}$ on $X$ by $d_{n}(z, y)=\max _{k=0, \ldots, n-1} d\left(f^{k}(z), f^{k}(y)\right)$. A set $\left\{z_{i}: i \in I\right\} \subset X$ is called $(n, \varepsilon)$-separated (with respect to $f$ ) if $d_{n}\left(z_{i}, z_{j}\right)>\varepsilon$ for all $z_{i}, z_{j}$ with $z_{i} \neq z_{j}$. For all $\varepsilon>0$ and $n \in \mathbb{N}$ fix a maximal (with respect to the inclusion) $(n, \varepsilon)$-separated set $F_{n}(\varepsilon)$. The topological pressure (with respect to $\left.f \mid X\right)$ is a map $P_{\text {top }}(f \mid X, \cdot): C(X, \mathbb{R}) \rightarrow \mathbb{R}$ defined by

$$
P_{\text {top }}(f \mid X, \varphi)=\lim _{\varepsilon \rightarrow 0} \limsup _{n \rightarrow \infty} \frac{1}{n} \log \left(\sum_{z \in F_{n}(\varepsilon)} \exp S_{n} \varphi(z)\right),
$$

where

$$
S_{n} \varphi(z)=\sum_{k=0}^{n-1} \varphi\left(f^{k}(z)\right) .
$$

The topological entropy of $f$ is defined by $h_{\text {top }}(f \mid X)=P_{\text {top }}(f \mid X, 0)$. For simplicity we write $P_{\text {top }}(\varphi)$ if there is no confusion about $f$ and $X$. Note that the definition of $P_{\mathrm{top}}(\varphi)$ does not depend on the choice of the sets $F_{n}(\varepsilon)$ (see [15]). The topological pressure satisfies the following variational principle:

$$
P_{\text {top }}(\varphi)=\sup _{\nu \in \mathcal{M}}\left(h_{\nu}(f)+\int_{\Lambda} \varphi d \nu\right) .
$$

Furthermore, the supremum in (10) can be taken only over all $\nu \in \mathcal{M}_{\mathrm{E}}$. We denote by $\operatorname{ES}(\varphi)$ the set of equilibrium states for $\varphi$, that is, of measures 
attaining the supremum in (10). We note that in general $\operatorname{ES}(\varphi)$ may be empty; however, if $f \in \mathcal{A}(X)$, then $\operatorname{ES}(\varphi)$ contains at least one (ergodic) measure. This follows from a result of Newhouse [10].

Next we introduce a pressure-like quantity by using the values of $\varphi$ on the periodic points in $X$. Let $\varphi \in C(X, \mathbb{R})$ and let $0<\alpha, 0<c \leq 1$. We define

$$
Q_{\mathrm{P}}(\varphi, \alpha, c, n)=\sum_{z \in \operatorname{Per}_{n}(\alpha, c)} \exp S_{n} \varphi(z)
$$

if $\operatorname{Per}_{n}(\alpha, c) \neq \emptyset$, and

$$
Q_{\mathrm{P}}(\varphi, \alpha, c, n)=\exp \left(n \min _{z \in X} \varphi(z)\right)
$$

otherwise. Furthermore, we define

$$
P_{\mathrm{P}}(\varphi, \alpha, c)=\limsup _{n \rightarrow \infty} \frac{1}{n} \log Q_{\mathrm{P}}(\varphi, \alpha, c, n) .
$$

It follows from the definition that if $\operatorname{Per}_{n}(\alpha, c) \neq \emptyset$ for some $n \in \mathbb{N}$ then this is true for infinitely many $n \in \mathbb{N}$. Therefore, in the case when $\operatorname{Per}_{n}(\alpha, c) \neq \emptyset$ for some $n \in \mathbb{N}$ then $P_{\mathrm{P}}(\varphi, \alpha, c)$ is entirely determined by the values of $\varphi$ on $\bigcup_{n \in \mathbb{N}} \operatorname{Per}_{n}(\alpha, c)$.

3. Pressure equals periodic point pressure. In this section we show for $f \in \mathcal{A}(X)$ and a rather general class of potentials that the topological pressure is entirely determined by the values of the potential on the repelling periodic points. More precisely, we prove the following theorem.

Theorem 2. Let $f \in \mathcal{A}(X)$ and let $\varphi \in C(X, \mathbb{R})$ be a Hölder continuous potential with $\alpha(\varphi)>0$.

(a) If $\alpha(\varphi)>0$ then for all $0<\alpha<\alpha(\varphi)$ we have

$$
P_{\text {top }}(\varphi)=\lim _{c \rightarrow 0} \limsup _{n \rightarrow \infty} \frac{1}{n} \log \left(\sum_{z \in \operatorname{Per}_{n}(\alpha, c)} \exp \left(\sum_{k=0}^{n-1} \varphi\left(f^{k}(z)\right)\right)\right) .
$$

(b) If (11) is true for some $\alpha>0$ then there exists an ergodic equilibrium state $\mu$ of $\varphi$ with $\chi(\mu) \geq \alpha$.

Rremark. Note that Theorem 2 immediately implies Theorem 1.

We delay the proof of Theorem 2 for a while and first prove some preliminary results.

Lemma 1. Let $f \in \mathcal{A}(X)$ and let $\Lambda$ be an invariant set on which $f$ is expanding. Let $\varphi \in C(\Lambda, \mathbb{R})$ be a Hölder continuous potential. Then

$$
\limsup _{n \rightarrow \infty} \frac{1}{n} \log \left(\sum_{z \in \operatorname{Per}_{n}(f \mid \Lambda)} \exp S_{n} \varphi(z)\right) \leq P_{\text {top }}(f \mid \Lambda, \varphi) .
$$


In particular, if $f \mid \Lambda$ is topologically mixing, then we have equality in (12), and the limit superior is in fact a limit.

Proof. Since $f \mid \Lambda$ is expanding it is expansive. Given any expansivity constant $\delta$, for every $n \in \mathbb{N}$ and every $0<\varepsilon \leq \delta$ the set $\operatorname{Per}_{n}(f \mid \Lambda)$ is $(n, \varepsilon)$-separated. Now, inequality (12) follows from the fact that the definition (8) can be replaced by the supremum taken over all $(n, \varepsilon)$-separated sets (see [15]). For the proof of the second statement we refer to [14, Chapter 7].

REMARK 1. The identity in (12) holds in the more general case of topologically mixing expanding maps (see [14, Chapter 7]). In particular, if $\Lambda$ is a repeller of a differentiable map $f$ such that $f \mid \Lambda$ is conjugate to a (one-sided) irreducible aperiodic subshift of finite type then (12) is an identity.

Proposition 1. Let $f \in \mathcal{A}(X)$ and let $\varphi \in C(X, \mathbb{R})$ be a continuous potential. Then for all $\mu \in \mathcal{M}_{\mathrm{E}}$ with $\chi(\mu)>0$ and for all $0<\alpha<\chi(\mu)$ we have

$$
h_{\mu}(f)+\int \varphi d \mu \leq \lim _{c \rightarrow 0} P_{\mathrm{P}}(\varphi, \alpha, c) .
$$

Proof. Consider $\mu \in \mathcal{M}_{\mathrm{E}}$ with $\chi(\mu)>0$, and fix $0<\alpha<\chi(\mu)$. Since $\chi(\mu)>0$, condition $(7)$ implies that $\operatorname{supp}(\mu) \subset X$ and thus the left hand side of (13) is well-defined. It is a consequence of Katok's theory [8] in its version for holomorphic endomorphisms developed by Przytycki and Urbański ([13, Chapter 9], see also [11]) that there exists a sequence of measures $\mu_{n} \in \mathcal{M}_{\mathrm{E}}$ supported on expanding sets $X_{n} \subset X$ such that

$$
h_{\mu}(f)+\int \varphi d \mu \leq \liminf _{n \rightarrow \infty} P_{\text {top }}\left(f \mid X_{n}, \varphi\right)
$$

and $\mu_{n} \rightarrow \mu$ with respect to the weak* topology. Moreover, for each $n \in \mathbb{N}$ there exist $m=m(n) \in \mathbb{N}$ and $s=s(n) \in \mathbb{N}$ such that $f^{m} \mid X_{n}$ is conjugate to the full shift on $s$ symbols. For every $0<\varepsilon<\chi(\mu)-\alpha$ there is a number $n=n(\varepsilon) \in \mathbb{N}$ such that

$$
h_{\mu}(f)+\int \varphi d \mu-\varepsilon \leq P_{\text {top }}\left(f \mid X_{n}, \varphi\right) .
$$

Moreover, there exists a number $c_{0}=c_{0}(n, \varepsilon)$ with $0<c_{0}(n) \leq 1$ such that for every periodic point $z \in X_{n}$ and every $k \in \mathbb{N}$ we have

$$
c_{0}^{-1} e^{k(\chi(\mu)-\varepsilon)} \leq\left|\left(f^{k}\right)^{\prime}(z)\right| \leq c_{0} e^{k(\chi(\mu)+\varepsilon)} .
$$

Note that (16) is a consequence of the construction of the sets $X_{n}$ in [13, Chapter 9.6]. This implies that

$$
\operatorname{Per}_{k}(f) \cap X_{n} \subset \operatorname{Per}_{k}\left(\alpha, c_{0}\right)
$$

for all $k \in \mathbb{N}$. Let $m, s \in \mathbb{N}$ be such that $f^{m} \mid X_{n}$ is topologically conjugate to the full shift on $s$ symbols. Since $m P_{\text {top }}\left(f \mid X_{n}, \varphi\right)=P_{\text {top }}\left(f^{m} \mid X_{n}, S_{m} \varphi\right)$ 
(see [15, Theorem 9.8]), we can conclude that

$$
h_{\mu}(f)+\int \varphi d \mu-\varepsilon \leq \frac{1}{m} P_{\mathrm{top}}\left(f^{m} \mid X_{n}, S_{m} \varphi\right) .
$$

Recall that $S_{m} \varphi(z)=\sum_{i=0}^{m-1} \varphi\left(f^{i}(z)\right)$. It now follows from Remark 1 and an elementary calculation that

$$
\begin{aligned}
h_{\mu}(f) & +\int \varphi d \mu-\varepsilon \\
& \leq \frac{1}{m} \lim _{k \rightarrow \infty} \frac{1}{k} \log \left(\sum_{z \in \operatorname{Per}_{m k}(f) \cap X_{n}} \exp \left(\sum_{i=0}^{k-1} S_{m} \varphi\left(f^{i m}(z)\right)\right)\right) \\
& \leq \lim _{k \rightarrow \infty} \frac{1}{k} \log \left(\sum_{z \in \operatorname{Per}_{k}(f) \cap X_{n}} \exp S_{k} \varphi(z)\right) .
\end{aligned}
$$

Combining (17) and (18) yields

$$
h_{\mu}(f)+\int \varphi d \mu-\varepsilon \leq \limsup _{k \rightarrow \infty} \frac{1}{k} \log \sum_{z \in \operatorname{Per}_{k}\left(\alpha, c_{0}\right)} \exp S_{k} \varphi(z) .
$$

Recall that by $(2)$ the map $c \mapsto P_{\mathrm{P}}(\varphi, \alpha, c)$ is non-decreasing as $c \rightarrow 0^{+}$. Since $\varepsilon>0$ is arbitrary the proof is complete.

We can now give the proof of Theorem 2.

Proof of Theorem 2. Let $0<\alpha$ and $0<c \leq 1$ be such that $\operatorname{Per}_{n}(\alpha, c) \neq \emptyset$ for some $n \in \mathbb{N}$. We first prove that

$$
P_{\mathrm{P}}(\varphi, \alpha, c) \leq \sup _{\nu}\left\{h_{\nu}(f)+\int_{\Lambda} \varphi d \nu\right\} \leq P_{\text {top }}(\varphi)
$$

where the supremum is taken over all $\nu \in \mathcal{M}_{\mathrm{E}}$ with $\alpha \leq \chi(\nu)$. Note that the supremum in (19) is not taken over the empty set. The right hand inequality in (19) is a consequence of the variational principle.

In order to prove the left hand inequality in (19) we define

$$
\Lambda=\Lambda_{\alpha, c}:=\overline{\bigcup_{n=1}^{\infty} \operatorname{Per}_{n}(\alpha, c)}
$$

It follows from a continuity argument that $f$ is repelling on $\Lambda$. Furthermore, for every $n \geq 1$ with $\operatorname{Per}_{n}(\alpha, c) \neq \emptyset$ we have

$$
\operatorname{Per}_{n}(f) \cap \Lambda=\operatorname{Per}_{n}(\alpha, c)
$$

Therefore, Lemma 1 implies that

$$
P_{\mathrm{P}}(\varphi, \alpha, c) \leq P_{\text {top }}(f \mid \Lambda, \varphi) .
$$

It follows from the variational principle that for every $\varepsilon>0$ there is a $\mu \in \mathcal{M}_{\mathrm{E}}$ 
which is supported in $\Lambda$ such that

$$
P_{\text {top }}(f \mid \Lambda, \varphi)-\varepsilon \leq h_{\mu}(f)+\int \varphi d \mu \leq P_{\text {top }}(f \mid \Lambda, \varphi) .
$$

Since $\mu$ is ergodic we have $\chi(z)=\chi(\mu)$ for $\mu$-almost every $z \in \Lambda$. It now follows from the continuity of $z \mapsto\left|f^{\prime}(z)\right|$ and the definition of $\operatorname{Per}_{n}(\alpha, c)$ that $\alpha \leq \chi(z)$ for all $z \in \Lambda$. We conclude that $\alpha \leq \chi(\mu)$. Therefore, the left hand inequality in (19) follows from (21) and (22).

Next, we prove that

$$
P_{\text {top }}(\varphi) \leq \lim _{c \rightarrow 0} P_{\mathrm{P}}(\varphi, \alpha, c) .
$$

Let $0<\alpha<\alpha(\varphi)$ and $0<\varepsilon<\alpha(\varphi)-\alpha$. It follows from the definition of $\alpha(\varphi)$ (see (5)) that there exist $\mu \in \mathcal{M}_{\mathrm{E}}$ with $\chi(\mu)>\alpha(\varphi)-\varepsilon>\alpha$ such that

$$
P_{\text {top }}(\varphi)=h_{\mu}(f)+\int \varphi d \mu .
$$

Therefore, Proposition 1 implies

$$
h_{\mu}(f)+\int \varphi d \mu \leq \lim _{c \rightarrow 0} P_{\mathrm{P}}(\varphi, \alpha, c) .
$$

Since $\varepsilon$ can be chosen arbitrarily small, (24) and (25) imply (23).

Finally, we prove (b). Let $\alpha>0$ be such that (11) holds. For $n \geq 1$ and $c>0$ with $\operatorname{Per}_{n}(\alpha, c) \neq \emptyset$ we define the measure $\sigma_{n}=\sigma_{n}(\alpha, c, \varphi) \in \mathcal{M}$ by

$$
\sigma_{n}=\frac{1}{\sum_{z \in \operatorname{Per}_{n}(\alpha, c)} \exp \left(S_{n} \varphi(z)\right)} \sum_{z \in \operatorname{Per}_{n}(\alpha, c)} \exp \left(S_{n} \varphi(z)\right) \delta_{z},
$$

where $\delta_{z}$ denotes the Dirac measure supported at $z$. Note that every measure $\sigma_{n}=\sigma_{n}(\alpha, c, \varphi)$ defined in (26) is in the convex hull of the set $\left\{\delta_{z}: z \in\right.$ $\left.\operatorname{Per}_{n}(\alpha, c)\right\}$. Consider a subsequence $\left(\sigma_{n_{k}}\right)_{k}$ converging to some measure $\mu_{\alpha, c}=\mu_{\alpha, c}(\varphi) \in \mathcal{M}$ in the weak ${ }^{*}$ topology. It follows that $\chi\left(\mu_{\alpha, c}\right) \geq \alpha$. Note that $f$ is expanding on $\Lambda_{\alpha, c}=\overline{\bigcup_{n=1}^{\infty} \operatorname{Per}_{n}(\alpha, c)}$. Thus, there exists an expansivity constant $\delta=\delta(\alpha, c)$ for $f \mid \Lambda_{\alpha, c}$. In particular, for every $n \in \mathbb{N}$ and every $0<\varepsilon \leq \delta$ the set $\operatorname{Per}_{n}(\alpha, c)$ is $(n, \varepsilon)$-separated. As in the proof of $[15$, Theorem 9.10] it follows that

$$
\limsup _{n \rightarrow \infty} \frac{1}{n} \log \sum_{x \in \operatorname{Per}_{n}(\alpha, c)} \exp \left(S_{n} \varphi(x)\right) \leq h_{\mu_{\alpha, c}}(f)+\int_{X} \varphi d \mu_{\alpha, c} .
$$

By construction, we have

$$
P_{\text {top }}(\varphi)=\lim _{c \rightarrow 0}\left(h_{\mu_{\alpha, c}}(f)+\int_{X} \varphi d \mu_{\alpha, c}\right) .
$$

As $c$ decreases to zero, there exists a subsequence $\left(\mu_{\alpha, c_{k}}\right)_{k}$ converging to some measure $\mu=\mu(\varphi) \in \mathcal{M}$ in the weak* topology. Using the upper semi- 
continuity of the entropy map and (28), we conclude that

$$
\lim _{c_{k} \rightarrow 0}\left(h_{\mu_{\alpha, c_{k}}}(f)+\int_{X} \varphi d \mu_{\alpha, c_{k}}\right)=h_{\mu}(f)+\int_{X} \varphi d \mu=P_{\text {top }}(\varphi) .
$$

It remains to show that $\chi(\mu) \geq \alpha$. This is trivial in case the sets $\Lambda_{\alpha, c_{k}}$ do not accumulate at critical points. To handle the case that the sets $\Lambda_{\alpha, c_{k}}$ possibly accumulate at a critical point $\gamma \in X$ we consider a decreasing sequence $\left(r_{i}\right)_{i}$ of positive numbers converging to 0 and a decreasing sequence of functions $\left(\phi_{i}\right)_{i}$ in $C(X, \mathbb{R})$ such that:

(i) $\phi_{i} \geq \log \left|f^{\prime}\right|$ and $\phi_{i}(z)=\log \left|f^{\prime}(z)\right|$ for all $z \in X \backslash B\left(\gamma, r_{i}\right)$.

(ii) $\phi_{i}(\gamma) \leq-i$.

In particular, $\phi_{i}$ converges pointwise to $\log \left|f^{\prime}\right|$. Fix $i \in \mathbb{N}$. Since $\mu_{\alpha, c_{k}}$ converges to $\mu$ in the weak* topology, we conclude that

$$
\int_{X} \phi_{i} d \mu=\lim _{k \rightarrow \infty} \int_{X} \phi_{i} d \mu_{\alpha, c_{k}} \geq \liminf _{k \rightarrow \infty} \chi\left(\mu_{\alpha, c_{k}}\right) \geq \alpha .
$$

It now follows from (3) and the monotone convergence theorem that

$$
\chi(\mu)=\lim _{i \rightarrow \infty} \int_{X} \phi_{i} d \mu \geq \alpha .
$$

One can choose $\mu$ to be ergodic by using an ergodic decomposition argument. The case when the sets $\Lambda_{\alpha, c_{k}}$ accumulate at finitely many critical points can be treated entirely analogously.

REMARK. We note that in the proof of Theorem 2 we have used similar techniques to those in our paper [6] in the case of $C^{2}$-diffeomorphisms, as well as ideas from [3] where the topological entropy (i.e. $\varphi=0$ ) of surface diffeomorphisms is studied.

\section{References}

[1] A. Arbieto and C. Matheus, Fast decay of correlations of equilibrium states of open classes of non-uniformly expanding maps and potentials, preprint, 2006.

[2] L. Carleson and T. Gamelin, Complex Dynamics, Springer, New York, 1993.

[3] Y. M. Chung and M. Hirayama, Topological entropy and periodic orbits of saddle type for surface diffeomorphisms, Hiroshima Math. J. 33 (2003), 189-195.

[4] M. Denker and M. Urbański, Ergodic theory of equilibrium states for rational maps, Nonlinearity 4 (1991), 103-134.

[5] A. Freire, A. Lopes, and R. Mañé, An invariant measure for rational maps, Bol. Soc. Brasil. Mat. 14 (1983), 45-62.

[6] K. Gelfert and C. Wolf, Topological pressure via periodic points, Trans. Amer. Math. Soc., to appear.

[7] F. Hofbauer and G. Keller, Equilibrium states for piecewise monotonic transformations, Ergodic Theory Dynam. Systems 2 (1982), 23-43. 
[8] A. Katok, Lyapunov exponents, entropy and periodic orbits for diffeomorphisms, Publ. Math. Inst. Hautes Études Sci. 51 (1980), 137-173.

[9] M. Lyubich, Entropy properties of rational endomorphisms of the Riemann sphere, Ergodic Theory Dynam. Systems 3 (1983), 351-385.

[10] S. Newhouse, Continuity properties of entropy, Ann. of Math. (2) 129 (1989), 215235.

[11] F. Przytycki, Expanding repellers in limit sets for iteration of holomorphic functions, Fund. Math. 186 (2005), 85-96.

[12] F. Przytycki, J. Rivera-Letelier, and S. Smirnov, Equality of pressures for rational functions, Ergodic Theory Dynam. Systems 24 (2004), 891-914.

[13] F. Przytycki and M. Urbański, Fractals in the Plane-the Ergodic Theory Methods, Cambridge Univ. Press, to appear.

[14] D. Ruelle, Thermodynamic Formalism, Cambridge Univ. Press, Cambridge, 2004.

[15] P. Walters, An Introduction to Ergodic Theory, Grad. Texts in Math. 79, Springer, New York, 1981.

Katrin Gelfert

Institute of Mathematics

Polish Academy of Sciences

Śniadeckich 8, P.O. Box 21

00-956 Warszawa, Poland

E-mail: gelfert@pks.mpg.de

http://www.pks.mpg.de/ gelfert/
Christian Wolf

Department of Mathematics

Wichita State University Wichita, KS 67260, U.S.A.

E-mail: cwolf@math.wichita.edu http://www.math.wichita.edu/ ${ }^{\sim}$ cwolf/

Received March 19, 2007;

received in final form April 5, 2007 